\title{
It's all fun and games until somebody gets hurt: using a scavenger hunt game to teach human factors to junior doctors
}

\author{
Author: Lucy Baxter
}

\section{Introduction}

Human factors are extremely important in healthcare. It is now a requirement that all junior doctors demonstrate understanding of human factors in medical error. ${ }^{1}$ Feedback from previous teaching to foundation doctors on human factors in South Tyneside NHS Foundation Trust included comments such as 'just not relevant to us at this level of our training'.

\section{Methods}

A session entitled 'Introduction to human factors' aimed to meet the outcome as expected in junior doctors' Foundation Programme curriculum: 'Describes the role of human factors in medical errors and takes steps to minimise these'.

The foundation doctors were divided into teams. They were given 15 minutes to collect items from a scavenger hunt list and complete bonus tasks. Points were awarded and deducted for adherence to task rules, items collected and tasks completed. Tasks required them to negotiate with education centre staff members, manage and prioritise their time, work as a team, and manage distractions. There were enough tasks and items to collect to create significant time pressures. Loud, repetitive music with a countdown timer was played. On completion they were asked to evaluate in pairs, then the wider group, what made the scavenger hunt difficult to complete, and what they did as a team that helped. As a group they then sorted their suggestions into two categories: human factors and non-technical skills. Definitions were given for both. At the end of the session, the foundation doctors were asked if they were to redo the scavenger hunt, what processes could improve their performance, and how these might be applicable to clinical work. They were asked to reflect on an error they had witnessed in clinical practice and apply the lessons they had learnt to this.

Results

Thirty-three foundation doctors attended the teaching over two sessions. Confidence in describing and identifying human factors, as well as taking steps to minimise them, increased by an average of $37 \%$. One hundred per cent of attendees felt that the session was relevant to their clinical practice and training. One hundred per cent listed the scavenger hunt as their favourite part of the session. Free text comments included: 'Really powerful and informative'; 'Interactive, fun, and backed up by relevant cases and information'; 'Turned a dry topic into something interesting and useful for our clinical practice'; and 'Good that we all had to get involved, learnt a lot more by the practical demonstration in the scavenger hunt that I have in previous sessions on the topic'.

\section{Conclusions}

Previously, junior trainees had found the topic of human factors dry, uninteresting and difficult to relate to their own practice. The scavenger hunt method allowed them to find the topic relatable and relevant. All attendees could describe the role of human factors in medical errors and take steps to minimise these, as per their curriculum, by the end of the session. A scavenger hunt game is a valuable method to introduce the topic of human factors and to demonstrate their practical application.

\section{Reference}

1 UK Foundation Programme. Curriculum. www.foundationprogramme.nhs.uk/content/curriculum-0 [Accessed 15 January 2019]. 